\title{
BMJ Open Impact of bed height on the biomechanics of healthcare professionals during chest compressions on the neonate: a descriptive pilot study
}

\author{
Jennifer Parent-Nichols (D) , ${ }^{1}$ Julia Perez, ${ }^{2}$ Brittany Witherell, ${ }^{3}$ Paula McWilliam, ${ }^{4}$ \\ Louis P Halamek, ${ }^{5}$ Nancy Kent, ${ }^{4}$ Wendy Varnum, ${ }^{4}$ Nicole K Yamada (i) ${ }^{5}$
}

To cite: Parent-Nichols J, Perez J, Witherell B, et al. Impact of bed height on the biomechanics of healthcare professionals during chest compressions on the neonate: a descriptive pilot study. BMJ Open 2021;11:e047666. doi:10.1136/ bmjopen-2020-047666

- Prepublication history for this paper is available online. To view these files, please visit the journal online (http://dx.doi. org/10.1136/bmjopen-2020047666).

Received 05 December 2020 Accepted 09 August 2021

\section{Check for updates}

(C) Author(s) (or their employer(s)) 2021. Re-use permitted under CC BY-NC. No commercial re-use. See rights and permissions. Published by BMJ.

For numbered affiliations see end of article.

\section{Correspondence to} Dr Jennifer Parent-Nichols; Jennifer.Parent_Nichols@Tufts. edu

\section{ABSTRACT}

Objectives The biomechanics of the healthcare

professionals (HCPs) performing the life-saving intervention of chest compressions in the neonatal population is poorly understood. The aim of this pilot study was to describe the variations in body position at a selfselected and a predetermined bed height during neonatal chest compressions. Measures of joint angles, time to postural sway and number of postural adjustments were chosen as indices for the stability of the HCP's position. Setting Data were collected at a simulation-based research centre in which the patient care environment was replicated.

Participants HCPs with varying roles working in the neonatal intensive care unit and holding a current Neonatal Resuscitation Program Provider certification were recruited for this study.

Interventions Fifteen HCPs performed two trials of chest compressions, each lasting $2 \mathrm{~min}$, at a predetermined bed height and a self-selected bed height. Trials were video recorded, capturing upper and lower body movements. Videos were analysed for time to postural sway and number of postural adjustments. Joint angles were measured at the start and end of each trial.

Results A statistically significant difference was found between the two bed height conditions for number of postural adjustments $(p=0.02)$. While not statistically significant, time postural sway was increased in the choice bed height condition (85s) compared with the predetermined bed height (45s). After $30 \mathrm{~s}$ of chest compressions, mean shoulder and knee angles were smaller for choice bed height $(p=0.03,95 \% \mathrm{Cl}$ Lower $=-12.14$, Upper $=-0.68$ and $p=0.05,95 \% \mathrm{Cl}$ Lower $=3.43$, Upper $=0.01$, respectively). After $1 \mathrm{~min}$ and $45 \mathrm{~s}$ of chest compressions, mean wrist angles were smaller in the choice bed height condition $(p=0.01,95 \% \mathrm{Cl}$ Lower $=-9.20$, Upper $=-1.22$ ), stride length decreased between the $30 \mathrm{~s}$ and $1 \mathrm{~min} 45 \mathrm{~s}$ marks of the chest compressions in the predetermined height condition $(p=0.02)$.

\section{INTRODUCTION}

Worldwide, approximately 3 million infants annually require resuscitation efforts at delivery that include chest compressions. ${ }^{1}$ The
Strengths and limitations of this study

- This is the first study to describe the biomechanics of healthcare professionals while performing neonatal chest compressions.

- Sample size was small, but demographic variation among the subject population was wide.

- Use of a simulated environment allowed for standardisation of all aspects of the study except for the variable (bed height) being studied.

- Order of trials may have magnified the effect of fatigue in the preset bed height trial.

need for extensive resuscitation, including chest compressions, in the neonatal intensive care unit (NICU) ranges from $0.1 \%$ for term to $15 \%$ for extremely preterm infants. ${ }^{1}$ To maximise the efficacy and consistency of chest compressions, equipment orientation and provider positioning must afford the healthcare professionals (HCPs) a biomechanical advantage where output effort is minimised. Failure to correctly perform chest compressions can lead to poor delivery of oxygen to vital organs and may result in lifelong morbidity or death. ${ }^{2}$

The biomechanics of the HCP providing the life-saving intervention of chest compressions in the neonatal population is poorly understood. Understanding the physical ergonomics of this technical procedure is useful in optimising the ability of HCPs to effectively deliver this intervention for the duration of a resuscitation. ${ }^{3}$ Current recommendations for chest compressions suggest that providers switch when they fatigue during chest compressions, but this does not account for the quality of chest compressions being performed. While studies have identified $2 \mathrm{~min}$ as the time point at which HCPs self-report fatigue when performing chest compressions, significant decreases in peak 
pressure have been found earlier than 2 min into resuscitation on a neonatal simulator. ${ }^{4-6}$ This finding suggests that objective measures might better determine the optimal time to switch providers during chest compressions.

The infant bed, either an isolette/incubator or radiant warmer, is central to all efforts by the neonatal resuscitation team. While the bed's primary features enable the stabilisation of the neonate, they are not easily adaptable to the anthropometric variations of the HCPs on resuscitation teams. In neonatal resuscitation, the provider stands at the side of the infant, arms held away from their body, with chest compressions provided with the thumbs. This technique requires that relative distance from the fulcrum to the point of external force application is increased, creating a less than optimal biomechanical position. ${ }^{7}$ Features that may encourage biomechanically advantageous positions for performing compressions are not easily accessible to all users. For instance, the pedals used to raise and lower both the isolette and radiant warmer bed height are typically located close to the centre post under the infant bed. Additionally, neither the bed width nor the lateral angle of the bed is adjustable to accommodate the variable stature, horizontal reach and abdominal depth of the HCPs on the resuscitation team. As a result, HCPs may require several postural adjustments to perform chest compressions.

Given the limitations of this integral piece of medical equipment, we sought to investigate the effects of one element of the resuscitation environment-namely, the height of the infant bed-on maintenance of a consistent HCP position as a surrogate for HCP effort during neonatal chest compressions. Previous studies in ergonomics have shown that when fatigue occurs during repetitive movements, as in the provision of chest compressions, changes in joint position and variability in movement become evident. Each of these postural changes may be considered as a task-specific control strategy intended to prolong performance. ${ }^{8}$ The aim of this pilot study was to begin to understand the effects of bed height on the biomechanics of HCPs performing neonatal chest compressions. Changes in joint angles, number of postural adjustments and time to anterior to posterior way were measured as indicators of procedural consistency.

\section{METHODS}

This study was conducted at a simulation-based research and training centre where highly realistic clinical scenarios are recreated in a physical environment that mimics the patient care environment.

Materials required for this study included one isolette (Atom Infant Incubator Dual i100, ATOM Medical, Wexford, PA, USA), one full-term neonatal patient simulator (SimNewB, Laerdal Medical; Stavanger, Norway), two iPads, tripods and floor grids. The application used for joint angle analysis, DrGoniometer, was uploaded to each iPad. Several studies support the validity and

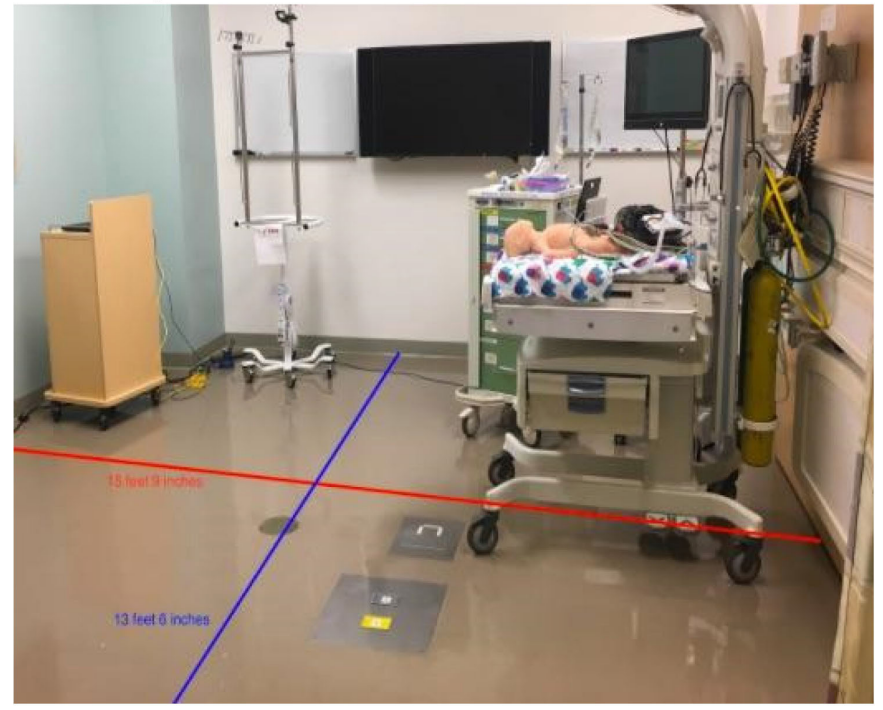

Figure 1 Room setup.

reliability for the use of this application for joint angle measurement. ${ }^{9-12}$ The setup of the room can be seen in figure 1.

In the present investigation, standardisation of the measurement protocol for the eight joint angles measured (cervical, thoracolumbar, shoulder, elbow, wrist, hip, knee and ankle) was performed. Universally accepted error in standard goniometric measurement is $\pm 5^{\circ} .{ }^{13-15}$ Joint angles for all eight joints were measured by each investigator from participant photographs using the DrGoniometer application. Measurements for each of the eight joints continued until all investigators' measurements were within $5^{\circ}$ of each other across five consecutive trials.

\section{Procedure}

\section{Patient and public involvement}

Participants were informed that findings will be shared on publication. No participants were directly involved in the design of this study.

\section{Recruitment}

A convenience sample of 15 participants from the NICU staff was recruited by electronic mail. Participants provided written informed consent prior to participating in the study. Criteria for participation in the study included working in an NICU and holding current Neonatal Resuscitation Program (NRP) Provider status.

\section{Procedural intervention}

The procedures for this study were as follows. First, demographic data were collected for the participants including the number of years working in the NICU, age, sex and clinical role. Anthropometric measurements obtained included knee height, hip height, elbow height, shoulder grip length, maximum vertical height, maximum forward reach, abdominal depth, elbow to fingertip, weight, eye dominance, hand dominance and shoe height. These measurements were taken according to the guidelines provided by Pheasant and Haslegrave. ${ }^{16}$ 
Next, participants were video recorded while administering two trials of chest compressions, each lasting $2 \mathrm{~min}$ in duration. A $3 \mathrm{~min}$ rest period was provided between trials. Trial 1 was conducted at the participant's selfselected ('choice') bed height and trial 2 was conducted at a predetermined bed height of $100 \mathrm{~cm}$. The minimum and maximum bed heights of the isolette used in this study are $80 \mathrm{~cm}$ and $120 \mathrm{~cm}$, respectively. The predetermined bed height was arbitrarily chosen as there is no standard bed height when the isolette is used in actual clinical care.

Chest compressions for both trials were performed on a full-term neonatal patient simulator. To simulate all components of neonatal cardiopulmonary resuscitation, participants were asked to pause after each cycle of three chest compressions to simulate the time that would be required to provide a breath. The neonatal patient simulator was maintained in a standardised position on the bed for all trials and participants all approached the task from the right side of the incubator which was closest to the entry point of the room. The two iPad cameras captured simultaneous videos of both events. One video captured the upper body; the other captured the lower body. It was anticipated that multiple videos would ensure that participant body position was adequately recorded during each trial regardless of the HCP position changes that might occur during each procedure. Finally, a 1-inch floor grid was affixed to the floor under participants' feet to facilitate measurements used in data analysis.

\section{Data analysis}

\section{Demographics and anthropometrics}

Demographic and anthropometric data for each participant can be found in table 1 .

\section{Postural changes}

To determine the ability of an HCP to maintain an assumed posture during chest compressions, two investigators independently viewed each participant's videos to determine the number of foot movements/foot position changes, referred to in this study as postural change, over the $2 \mathrm{~min}$ as well as the time from the initiation of chest compressions to the time the participant began to demonstrate anterior to posterior sway of the whole body during delivery of compressions. These measurements were performed for both the predetermined bed height and choice bed height trials. When a discrepancy in number of postural changes between investigators occurred, the third investigator reviewed the video and made the final determination.

\section{Joint angle changes}

To determine if joint angles changed for the participants from the beginning of the $2 \mathrm{~min}$ of chest compressions to the end for both bed height trials, screenshots were captured from each video at the $30 \mathrm{~s}$ and 1 min $45 \mathrm{~s}$ marks for comparison. The $30 \mathrm{~s}$ time point allowed sufficient time for the participant to establish a rhythm for chest
Table 1 Demographic and anthropometric data

\begin{tabular}{|c|c|}
\hline Demographic data & Counts \\
\hline \multirow[t]{4}{*}{ Age of participants } & $30-39(7)$ \\
\hline & $40-49(5)$ \\
\hline & $50-59(2)$ \\
\hline & $60-69(1)$ \\
\hline \multirow[t]{2}{*}{ Sex } & Female (12) \\
\hline & Male (3) \\
\hline \multirow[t]{5}{*}{ Clinical role } & Neonatology attending (7) \\
\hline & Neonatal nurse practitioner (4) \\
\hline & $\begin{array}{l}\text { Registered nurse transport } \\
\text { specialist (2) }\end{array}$ \\
\hline & Neonatology fellow (1) \\
\hline & Neonatal hospitalist (1) \\
\hline \multirow{7}{*}{$\begin{array}{l}\text { Years of work in the } \\
\text { neonatal intensive care unit }\end{array}$} & $0-5$ years $(2)$ \\
\hline & $6-10$ years $(3)$ \\
\hline & $11-15$ years $(3)$ \\
\hline & $16-20$ years $(3)$ \\
\hline & $21-25$ years $(2)$ \\
\hline & $26-30$ years $(0)$ \\
\hline & $31-35$ years $(2)$ \\
\hline \multirow[t]{2}{*}{ Hand dominance } & Right (11) \\
\hline & Left (4) \\
\hline Anthropometric data & Average/SD \\
\hline Total stature (height +shoe) & $172 \mathrm{~cm}(\mathrm{SD} \pm 11.82)$ \\
\hline Grip strength & $29.46 \mathrm{~kg}(\mathrm{SD} \pm 2.27)$ \\
\hline Pinch strength & $6.96 \mathrm{~kg}(\mathrm{SD} \pm 0.68)$ \\
\hline Max forward grip & $69.4267 \mathrm{~cm}(\mathrm{SD} \pm 8.0058)$ \\
\hline Abdominal depth & $20.87 \mathrm{~cm}(\mathrm{SD} \pm 4.4051)$ \\
\hline
\end{tabular}

compressions and the $1 \mathrm{~min} 45 \mathrm{~s}$ time point approached the end of the trial without the participant anticipating completion. Joint angle measurements were calculated from these screenshots using the DrGoniometer application for the following joints for both trials: cervical, shoulder, elbow, wrist, thoracolumbar, hip, knee and ankle. Gold standard landmarks described in Kendall et al were used for all measurements. ${ }^{17}$

The biplanar nature of still photos necessitated that the cervical, thoracolumbar and wrist measurement procedures be adjusted to obtain standardised measurements. Landmarks for these measurements can be found in table 2.

For all joint angle measurements, two investigators separately calculated measurements and the mean of these measures was calculated and used for statistical analysis. If a greater than a five-degree difference occurred between investigators, a third investigator measured the angle. In this case, the mean of the three measurements was used for statistical analysis. Using the 1-inch grid on the floor, stance width and stride length were recorded from the 
Table 2 Non-standard goniometric measurement landmarks

\begin{tabular}{ll}
\hline Joint & Landmark \\
\hline Cervical & $\begin{array}{l}\text { Fulcrum: external auditory meatus } \\
\text { Moving arm: base of nose } \\
\text { Stationary arm: perpendicular to crown of } \\
\text { the head }\end{array}$ \\
Thoracolumbar & $\begin{array}{l}\text { Fulcrum: centred between the anterior } \\
\text { superior iliac spine (ASIS) and posterior } \\
\end{array}$ \\
& $\begin{array}{l}\text { Superior iliac spine (PSIS) } \\
\text { the angle of the ASIS and PSIS }\end{array}$ \\
& Stationary arm: in line with radius \\
& Fulcrum: radial styloid \\
Wrist & Moving arm: in line with second metacarpal \\
& Stationary arm: in line with radius \\
\hline
\end{tabular}

great toe of the right foot to the great toe of the left foot using the still photos.

\section{Statistical analysis}

Postural changes

To answer the primary question of whether bed height influenced consistency of positioning by HCPs, data for the number of postural adjustments and time to anterior to posterior postural sway during chest compressions were analysed at both a standard bed height of $100 \mathrm{~cm}$ and the HCP's choice bed height. A Shapiro-Wilk's test of normality was performed to determine if these data were normally distributed. If data were normally distributed, a paired samples t-test was performed. In the case where data were not normally distributed, a Wilcoxon signed-rank test was performed. A paired samples t-test was performed to analyse time to anterior to posterior postural sway. A Wilcoxon signed-rank test was run on the number of postural adjustments as counts were found to be not normally distributed.

\section{Joint angle changes}

Differences in joint angle measurements between bed height conditions were examined at the $30 \mathrm{~s}$ and $1 \mathrm{~min}$ $45 \mathrm{~s}$ marks. As two independent researchers measured each joint, an intraclass correlation $(3,1)$ was conducted for each joint angle measurement to determine the interrater reliability of all joint measurements. A ShapiroWilk's test of normality was performed on the nine angles at $30 \mathrm{~s}$ and $1 \mathrm{~min} 45 \mathrm{~s}$ for both trials to ensure data analysis could be performed with a paired samples t-test.

A Wilcoxon signed-rank test was conducted on nonnormal data of cervical and ankle angle in the choice bed height trial and ankle angle and stride length at the predetermined bed height at the $30 \mathrm{~s}$ mark. A Wilcoxon signed-rank test was also used for knee and ankle joints and stride length data int the choice bed height trial and for the ankle joint and stride length in the predetermined bed height trial at the 1 min $45 \mathrm{~s}$ mark. A paired samples t-test was used on the remaining measurements as data were found to be normally distributed.

Differences in joint angles at the $30 \mathrm{~s}$ and $1 \mathrm{~min} 45 \mathrm{~s}$ mark were examined within each bed height trial. Cervical and ankle joint angles at the $30 \mathrm{~s}$ mark and the knee and ankle joint at the 1 min $45 \mathrm{~s}$ mark were not normally distributed in the choice bed height trial. Ankle joint angle and stride length at the $30 \mathrm{~s}$ and $1 \mathrm{~min} 45 \mathrm{~s}$ mark were also not normally distributed for the predetermined bed height trial. For these data, a Wilcoxon signed-rank test was conducted. A paired samples t-test was performed for the remaining data that demonstrated a normal distribution.

Additionally, a Pearson correlation was performed to determine if there was a relationship between participant total stature (participant height plus shoe) and selected bed height.

All data analyses were performed using SPSS Statistics V.25. For all statistical analyses, the alpha level was set at 0.05 .

\section{RESULTS}

\section{Demographic and anthropometric data}

Data from all 15 participants, 12 women and 3 men, were included in this analysis. Clinical roles of the participants were neonatology attending physicians (7), neonatal nurse practitioners (4), neonatal nurse transport specialists (2), neonatology hospitalist (1) and neonatology fellow (1). Of the 15 participants, two had worked in the NICU for $0-5$ years, three for 11-15 years, three from 16 to 20 years, two from 21 to 25 years and two from 31 to 35 years. The mean age of participants was 42 years $(\mathrm{SD}=10.25)$. The means and SD of participant anthropometric attributes were maximal forward grip $69 \mathrm{~cm}$ (SD $8.01)$, abdominal depth $21 \mathrm{~cm}(\mathrm{SD}=4.41)$ and total stature $172 \mathrm{~cm}(\mathrm{SD}=11.82)$.

\section{Postural changes}

Results of the Wilcoxon signed-rank test revealed a statistically significant difference in number of postural adjustments $(\mathrm{p}=0.02)$ between the two bed height trials. Median times to anterior to posterior sway were longer in the choice bed height condition (85s) than in the predetermined bed height condition (45s). However, this difference was not statistically significant $(\mathrm{p}=0.08)$. Figure 2 shows a decreased number of postural adjustments made by the participants in the choice bed height trial compared with the predetermined bed height trial.

Figure 3 shows increased time to anterior to posterior postural sway during chest compressions in the choice bed height trial compared with the predetermined bed height trial.

\section{Joint angle change}

A two-way mixed interclass correlation $(3,1)$ showed good to excellent reliability (ICC 0.87 to 0.99 ) between the two independent researchers for all measured joint angles. Upper and lower bounds for the 95\% CI were 0.63 and 


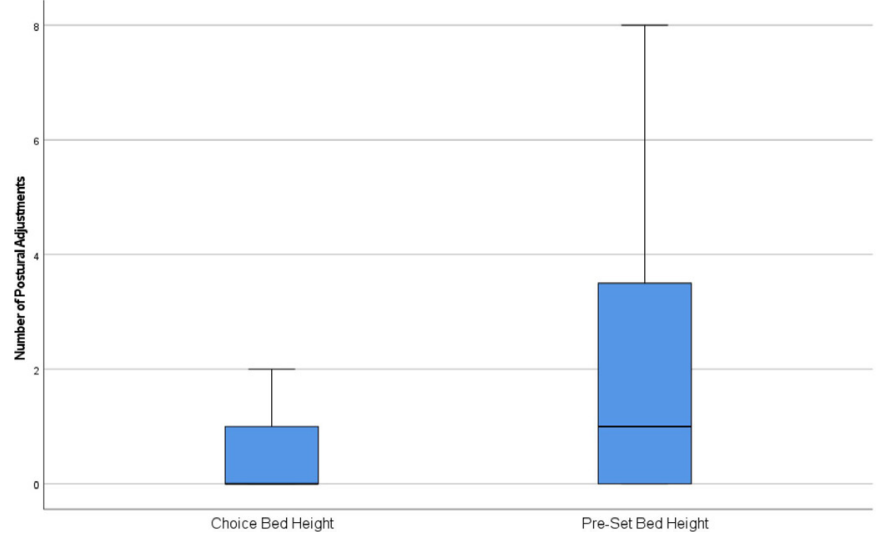

Figure 2 Number of postural adjustments by chest compression choice to pre-set bed height.

0.99 , respectively. The ICC for each measurement can be found in table 3 .

Between bed height analysis of joint angles: $30 \mathrm{~s}$

At the $30 \mathrm{~s}$ mark, mean shoulder and knee angles were smaller for the choice bed height trial compared with the predetermined bed height trial $(\mathrm{p}=0.03,95 \% \mathrm{CI}$ Lower $=-12.14$, Upper $=-0.68$ and $\quad \mathrm{p}=0.05, \quad 95 \% \mathrm{CI}$ Lower $=-3.43$, Upper $=0.01$, respectively).

\section{Between bed height analysis of joint angles: 1 min $45 \mathrm{~s}$}

At the $1 \mathrm{~min} 45 \mathrm{~s}$ mark, mean wrist angles were smaller for the choice bed height trial compared with the predetermined bed height trial $(\mathrm{p}=0.01,95 \%$ CI Lower -9.20 , Upper-1.22).

\section{Within bed height analysis at $30 \mathrm{~s}$ and $1 \mathrm{~min} 45 \mathrm{~s}$}

No statistically significant differences were found in any joint angle between the $30 \mathrm{~s}$ to $1 \mathrm{~min} 45 \mathrm{~s}$ trials for the choice bed height or the predetermined bed height trial. Stride length was shorter at the 1 min $45 \mathrm{~s}$ mark than at the $30 \mathrm{~s}$ mark in the predetermined bed height trial $(\mathrm{p}=0.02)$.

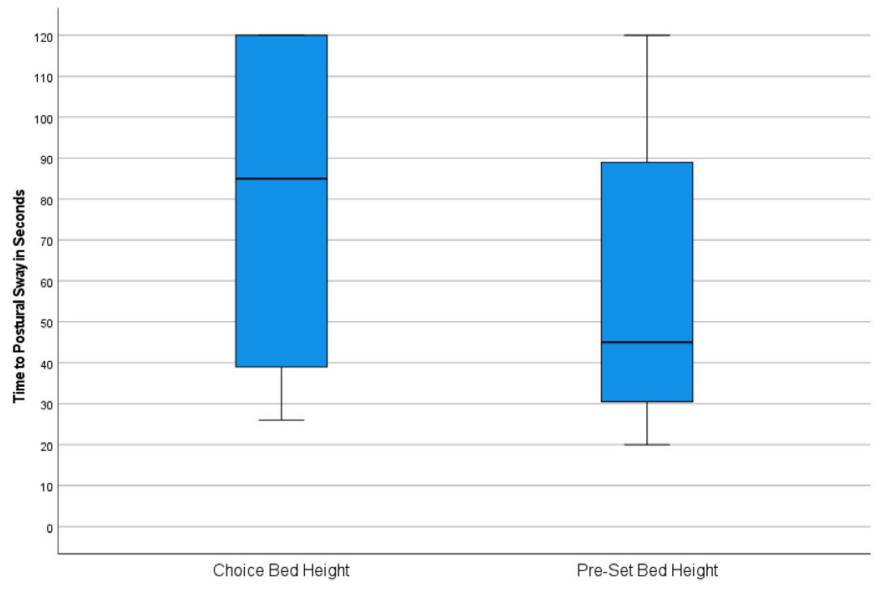

Figure 3 Time to anterior to postural sway at choice and predetermined bed height.
Table 3 Intraclass correlation coefficients for joint measurements

Intraclass correlation coefficient $(3,1)$

\begin{tabular}{ll}
$\begin{array}{l}\text { Upper and lower bounds for } \mathrm{Cl} \text { : } \\
0.63 \text { to } 0.99\end{array}$ \\
\hline Cervical & 0.94 \\
\hline Thoracolumbar & 0.89 \\
Shoulder & 0.98 \\
\hline Elbow & 0.99 \\
\hline Wrist & 0.94 \\
\hline Hip & 0.96 \\
Knee & 0.87 \\
Ankle & 0.99 \\
Stride width & 0.97 \\
Stride length & 0.99
\end{tabular}

Bed height to stature relationship

A statistically significant relationship was found between participant height and selected bed height $(p=0.03)$ with $30.6 \%$ of variance ascribed to participant height. Figure 4 shows the relationship between the bed height selected and participant height.

\section{DISCUSSION}

This study aimed to describe the postural changes observed over two trials of neonatal chest compressions at two different bed heights, one chosen by the participant and one predetermined. Differences in number of postural adjustments and time to postural sway were found between the two trials. Further, differences in joint angles, knee and shoulder at $30 \mathrm{~s}$ and the wrist at $1 \mathrm{~min}$ $45 \mathrm{~s}$ were seen between the two trials. A narrower stride length was evident in the predetermined bed height trial.

Chest compressions are a critical technical procedure in cardiopulmonary resuscitation. Ergonomic studies of chest compressions in the field of neonatal resuscitation have focused either on neonatal physiology or assessment

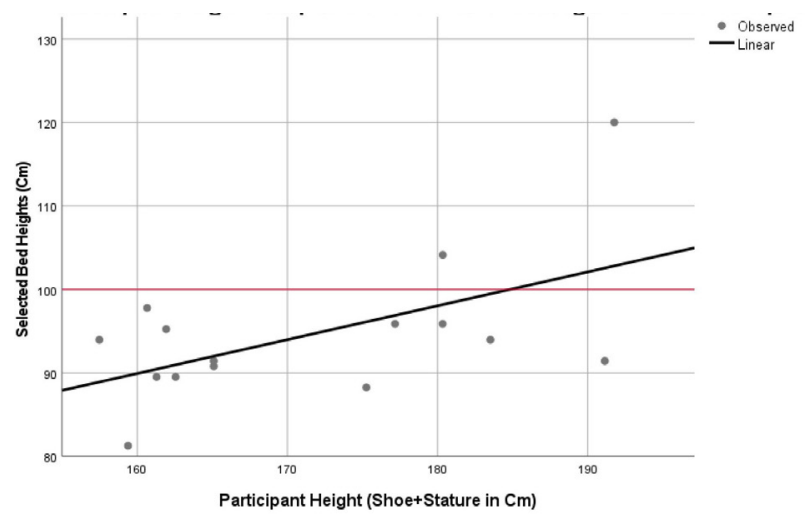

Figure 4 Participant height compared with selected bed height for chest compressions. 
of techniques that most effectively generate adequate coronary perfusion pressure for return of spontaneous circulation. ${ }^{5}$ Few studies examine the effects of ergonomics or position on the ability of HCPs to initiate and sustain effective chest compressions. To date, most studies examining ergonomics of resuscitation have focused on adult resuscitation. In these adult studies, chest compressions were shown to be more effective and required less perceived exertion when the HCP was positioned in closest proximity to the adult patient and at a bed height of their choosing. ${ }^{18-20}$ As the approach to neonatal resuscitation differs from that of the adult, it is possible that the efficacy and consistency of performance over time could differ as well.

Maintenance of a position of biomechanical advantage may enhance performance over the duration of the task. Changes in joint position and variability in movement have been identified as means to alter task-specific control strategies to allow for motor task completetion. ${ }^{821}$ During physically demanding, high intensity tasks such as providing chest compressions, individuals may alter motor strategies to continue that task for a required time. The interjoint connections of the human body allow for compensatory postural changes aimed at task completion. $^{22} 23$

Outcomes for other studies investigating performance of repetitive activities indicate that postural adaptations are typically made in response to local and global fatigue in the body. In these studies, localised fatigue resulting from a repetitive motor task resulted in increased global movement to complete a task. In short, when individuals fatigued during a motor task, they altered their initial motor strategies to complete the motor task. ${ }^{22}{ }^{23}$ In this present study, participants made postural adjustments more frequently and sooner in the cycle at the predetermined bed height compared with the choice bed height. The lack of a finding of statistical significance for the time to anterior to posterior sway may be explained by the small sample size for this pilot. A larger sample size may provide greater insight. Nevertheless, these differences suggest that the predetermined bed height was less biomechanically advantageous as participants required more and earlier alterations in their positions to accomplish the end goal of performing chest compressions on a neonatal patient simulator for $2 \mathrm{~min}$ at the predetermined bed height.

A study of the impact of muscle fatigue on movement strategy found that interjoint coordination decreased with fatigue. Over the course of a task, participants attempted to limit the $\mathrm{df}$ available at their joints and adopted a more rigid movement strategy. ${ }^{23}$ In the current study, participants may have attempted to control their df initially at proximal joints and, as the trial progressed, at more distal joints. When comparing chest compressions between bed height conditions, a statistically significant difference in joint angles was noted at the $30 \mathrm{~s}$ mark in the larger joints of the knee and shoulder and then at the 1 min $45 \mathrm{~s}$ mark in the smaller joint of the wrist. Joint angle measures were smaller in the choice bed height condition, indicating a less flexed, more open position of those joints. Additionally, participants adopted a narrower stride length in the predetermined bed height condition. A narrowed stance may have allowed participants to increase their leverage in this condition. In contrast, no statistically significant differences in stride length were found in the choice bed height condition, indicating that this adaptation was not required when participants could select their preferred bed height. When HCP's can choose the height of the bed, they may not require adaptations in body position that garner a biomechanical advantage for the completion of chest compressions because the bed height itself allows for a more advantageous approach.

Lastly, only the change in joint angle at the ankle in the choice bed height condition was predictive of number of postural adjustments. No joint angle change for either condition was predictive of time to anterior to posterior postural sway. It is possible that participants made postural adaptations across several joints throughout the trials to continue to execute necessary chest compressions.

\section{Considerations}

There are several considerations that should be made when interpreting the results of this study. Each participant was prompted to perform chest compressions at their preferred bed height first and the predetermined bed height second. This order may have led to participant fatigue, magnifying any effects found in the subsequent predetermined height trial. Attempts to mitigate this effect were made by requiring each participant to take a 3 min break between trials.

Participants were not studied when performing chest compressions from the head of the bed, which recent NRP guidelines now recommend. ${ }^{24}$ This position may provide mechanical advantages that we were not able to examine in this study. However, chest compressions are still commonly initiated with the HCP standing at the side of the bed and the findings reported here are especially relevant to the first round of chest compressions performed during neonatal resuscitation.

The neonatal patient simulator used in this study has a thoracic circumference of 15 inches, which is larger than the average newborn thoracic circumference of 12-13 inches. As a result, the adjustments made by participants in this study may not exactly replicate those made during the care of term or preterm newborns during actual clinical care.

The sample size of this study was small. However, statistically significant results were found and demographic variation among the participant population was wide, enhancing the generalisability to HCPs who perform chest compressions in neonatal patients.

Goniometric measurements of joint angles were within an acceptable variation of five degrees. No joint angle was measured by fewer than two independent investigators. Use of the simulation lab allowed for standardisation of 
many aspects of the research environment, limiting the number of confounding variables.

This study is the first to describe biomechanics related to the provision of chest compressions in the neonate. Future investigations should aim to identify whether alterations in body mechanics during chest compressions may serve as visual indicators of decreased compression efficacy and whether the ability to choose a bed height may improve compression consistency and efficacy.

\section{CONCLUSION}

This study aimed to describe the biomechanics of healthcare providers during two trials of chest compressions at two different bed heights. The findings of this study indicate that HCPs change body position less frequently and demonstrate anterior to posterior postural sway later during chest compressions in the trial where the bed height was chosen. Changing of body position or initiation of anterior to posterior postural sway may indicate the need of the HCP to alter their approach to continue with resuscitation efforts. The ability to maintain a consistent position for an HCP may influence the efficacy and consistency in the provision of chest compressions for neonates. Altering the height of the bed to accommodate HCP height may provide a position of biomechanical advantage.

\section{Author affiliations}

${ }^{1}$ Doctor of Physical Therapy Program, Tufts University School of Medicine, Boston, Massachusetts, USA

${ }^{2}$ Physical Therapy, The Spine and Health Center of Montvale, Montvale, New Jersey, USA

${ }^{3}$ Physical Therapy, Elliot Physical Therapy, Dorchester, Massachusetts, USA

${ }^{4}$ Nursing, Franklin Pierce University, Manchester, New Hampshire, USA

${ }^{5}$ Division of Neonatal and Developmental Medicine, Department of Pediatrics,

Stanford University School of Medicine, Stanford, California, USA

\section{Twitter Nicole K Yamada @StanfordCAPE}

Acknowledgements We express our gratitude to the Center for Advanced Pediatric and Perinatal Education at Stanford University.

Contributors JP-N contributed to study design, execution, data analysis and manuscript development and submission. JP and BW assisted with study design, execution, data analysis and manuscript preparation. PM ensured funding for this project and assisted with the study design, execution, data analysis and manuscript preparation. LH served as a consultant for this project and was directly involved in the development of this manuscript. NK assisted with statistical analysis. WV provided data collection and entry. NKY served as a mentor and collaborator and was directly involved with all aspects of the study, including study design, execution and development of this manuscript.

Funding This research was funded by IDeA Network of Biomedical Research Excellence (P20GM103506) and the Center for Advanced Pediatric and Perinatal Education (CAPE), Simulation-based Healthcare Training and Research Services endowment (No grant number).

Competing interests None declared.

Patient and public involvement Patients and/or the public were not involved in the design, or conduct, or reporting, or dissemination plans of this research.

Patient consent for publication Not required.

Ethics approval Institutional Review Board approval was received from both participating universities. Franklin Pierce University: \#PW8162018. Stanford University eProtocol \#: 65681. Participants provided written informed consent prior to participating in the study.
Provenance and peer review Not commissioned; externally peer reviewed.

Data availability statement Data are available upon reasonable request.

Open access This is an open access article distributed in accordance with the Creative Commons Attribution Non Commercial (CC BY-NC 4.0) license, which permits others to distribute, remix, adapt, build upon this work non-commercially, and license their derivative works on different terms, provided the original work is properly cited, appropriate credit is given, any changes made indicated, and the use is non-commercial. See: http://creativecommons.org/licenses/by-nc/4.0/.

ORCID iDs

Jennifer Parent-Nichols http://orcid.org/0000-0002-1346-6462

Nicole K Yamada http://orcid.org/0000-0001-6800-8306

\section{REFERENCES}

1 Garcia-Hidalgo C, Schmölzer GM. Chest compressions in the delivery room. Children 2019;6:4.

2 Chadha IA. Neonatal resuscitation: current issues. Indian J Anaesth 2010;54:428.

3 Yamada NK, Fuerch JH, Halamek LP. Ergonomic challenges inherent in neonatal resuscitation. Children 2019;6:74.

4 Li ES, Cheung P-Y, O'Reilly M, et al. Rescuer fatigue during simulated neonatal cardiopulmonary resuscitation. J Perinatol 2015;35:142-5

5 Douvanas A, Koulouglioti C, Kalafati M. A comparison between the two methods of chest compression in infant and neonatal resuscitation. A review according to 2010 CPR guidelines. J Matern Fetal Neonatal Med 2018;31:805-16.

6 Enriquez D, Firenze L, Fernández Díaz J, et al. Changes in the depth of chest compressions during cardiopulmonary resuscitation in a pediatric simulator. Arch Argent Pediatr 2018;116:e730-5.

7 Lu T-W, Chang C-F. Biomechanics of human movement and its clinical applications. Kaohsiung J Med Sci 2012;28:S13-25.

8 Fuller JR, Fung J, Côté JN. Time-dependent adaptations to posture and movement characteristics during the development of repetitive reaching induced fatigue. Exp Brain Res 2011;211:133-43.

9 Crasto JA, Sayari AJ, Gray RR-L, et al. Comparative analysis of photograph-based clinical goniometry to standard techniques. Hand 2015;10:248-53.

10 Behnoush B, Tavakoli N, Bazmi E, et al. Smartphone and universal goniometer for measurement of elbow joint motions: a comparative study. Asian J Sports Med 2016;7:e30668.

11 Otter SJ, Agalliu B, Baer N, et al. The reliability of a smartphone goniometer application compared with a traditional goniometer for measuring first metatarsophalangeal joint dorsiflexion. J Foot Ankle Res 2015;8:30.

12 Milani P, Coccetta CA, Rabini A. Mobile smartphone applications for body position measurement in rehabilitation: a review of goniometric tools. PM\&R 2014;6:1038-43.

13 McVeigh KH, Murray PM, Heckman MG, et al. Accuracy and validity of goniometer and visual assessments of angular joint positions of the hand and wrist. J Hand Surg Am 2016;41:e21-35.

14 Norkin CC, White DJ. Measurement of joint motion: a guide to goniometry. FA Davis 2016.

15 Boone DC, Azen SP, Lin CM, et al. Reliability of goniometric measurements. Phys Ther 1978;58:1355-60.

16 Pheasant S, Haslegrave CM. Bodyspace: anthropometry, ergonomics and the design of work. CRC press, 2005.

17 Kendall FP, McCreary EK, Provance PG, et al. Muscles: testing and function, with posture and pain (Kendall, muscles). Lippincott Williams and Wilkins, 2005.

18 Cho J, Oh JH, Park YS, et al. Effects of bed height on the performance of chest compressions. Emerg Med J 2009;26:807-10.

$19 \mathrm{MS} \mathrm{H}$, Chow DH. Effects of kneeling posture on chest compression during cardiopulmonary resuscitation in males. J Clin Nurs 2018;27:777-83.

20 Zhou XL, Sheng LP, Wang J, et al. Effect of bed width on the quality of compressions in simulated resuscitation: a randomized crossover manikin study. Am J Emerg Med 2016;34:2272-6.

21 Cowley JC, Dingwell JB, Gates DH. Effects of local and widespread muscle fatigue on movement timing. Exp Brain Res 2014;232:3939-48.

22 Srinivasan D, Mathiassen SE. Motor variability--an important issue in occupational life. Work 2012;41:2527-34.

23 Cowley JC, Gates DH. Inter-joint coordination changes during and after muscle fatigue. Hum Mov Sci 2017;56:109-18.

24 Weiner GM, Zaichkin J. Textbook of neonatal resuscitation. 7th edn Elk Grove Village, IL: American Academy of Pediatrics, 2016. 